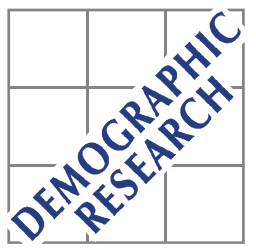

Demographic Research a free, expedited, online journal of peer-reviewed research and commentary in the population sciences published by the Max Planck Institute for Demographic Research Konrad-Zuse Str. 1, D-18057 Rostock · GERMANY www.demographic-research.org

DEMOGRAPHIC RESEARCH

VOLUME 13, ARTICLE 3, PAGES 63-82

PUBLISHED 19 AUGUST 2005

http://www.demographic-research.org/Volumes/Vol13/3/

DOI: $10.4054 /$ DemRes.2005.13.3

Research Article

Age-specific contributions to changes in the period and cohort life expectancy

\title{
Vladimir Canudas-Romo
}

Robert Schoen

(C) 2005 Max-Planck-Gesellschaft. 


\section{Table of Contents}

1 Introduction $\quad 64$

$2 \quad$ Period and cohort life expectancy $\quad 64$

$3 \quad$ Period and cohort life expectancy age-decomposition 66

$4 \quad$ Changes in period and cohort life expectancy $\quad 67$

$\begin{array}{lll}4.1 & \text { Period and cohort models of mortality } & 67\end{array}$

4.2 Trends, gaps and lags involving period and cohort life expectancies $\quad 69$

4.3 Age-specific contributions to changes in period 72 and cohort life expectancy

$5 \quad$ Examining period and cohort mortality in Sweden 76

$\begin{array}{lll}6 & \text { Conclusions } & 79\end{array}$

$\begin{array}{lll}7 & \text { Acknowledgments } & 79\end{array}$

$\begin{array}{ll}\text { References } & 80\end{array}$ 


\title{
Age-specific contributions to changes in the period and cohort life expectancy
}

\author{
Vladimir Canudas-Romo
}

\author{
Robert Schoen
}

\begin{abstract}
Period life expectancy has increased more slowly than its cohort counterpart. This paper explores the differences between life expectancies at a given time (the gap) and the time required for period life expectancy to reach the current level of cohort life expectancy (the lag). Additionally, to understand the disparity between the two life expectancies we identify and compare age-specific contributions to change in life expectancy. Using mortality models and historical data for Sweden, we examine the effect of mortality changes over time. Our results indicate that the widening of the gap between the two life expectancies is primarily a consequence of the dramatic mortality decline at older ages that occurred during the twentieth century. These results imply that the divergence between the two measures is likely to become even greater in the future as reductions in deaths are concentrated at older ages.
\end{abstract}




\section{Introduction}

Cohort life tables follow the mortality of a given birth cohort over its life course. Some industrialized countries have uninterrupted data spanning several centuries that allow such analyses. Period life tables, which require data from only one year, can depict the implications of recent rates. Period life expectancies have been used for comparisons over time and across populations as a matter of accepted practice. However, Bongaarts and Feeney (2002) suggested that the conventional period life expectancy is an inaccurate measure for countries with low mortality. Regarding the industrialized world, substantial gaps and lags between period and cohort life expectancies have been shown by Goldstein and Wachter (2005), which stimulated interest in the subject. As defined by these authors, a gap tells how much period life expectancy at a given time is less than the life expectancy of the cohort born in that year. The lag (or forward lag) is the time required for period life expectancy to reach the current level of cohort life expectancy. In nineteenth century Sweden, the two life expectancies had similar values, but over time the disparity between them grew as increases in cohort life expectancies outstripped increases in period life expectancies.

Recognizing the mechanisms that relate period and cohort perspectives on the average length of life contributes to the analysis of past, current and future trends in mortality. In this paper we present some simple relations between the gaps and lags arising from the distinct levels of period and cohort life expectancies. We then use decomposition techniques to study the age-specific contributions to changes in life expectancy that drive aggregate trends.

In the next section we bring definitions relevant to our study of mortality, followed with an examination of changes over time in life expectancies. Then, age-specific contributions to period and cohort life expectancies are calculated and compared using an age-decomposition of life expectancy. Mortality models are used to simplify the relations between gaps and lags in life expectancies and to demonstrate how period and cohort life expectancies change under different mortality patterns. Finally, applications to the mortality experience of nineteenth and twentieth century Sweden are provided.

\section{Period and cohort life expectancy}

The most commonly known measures of mortality are the period and cohort life expectancy. In the life table context, period life expectancy at age $a$ and time $t$ is calculated as the person-years lived above age $a$ divided by the number surviving to age $a$. For example, the period life expectancy at birth at time $t$ can be expressed as 


$$
e_{p}(0, t)=\frac{\int_{0}^{\omega} \ell_{p}(a, t) d a}{\ell_{p}(0, t)},
$$

where $\ell_{p}(a, t)$ is the period life table survivorship function to age $a$ under the rates at time $t$, and $\varpi$ is the highest age attained. If the radix of the table is one, i.e. $\ell_{p}(0, t)=1$, then $\ell_{p}(a, t)$ is the period life table probability of surviving from birth to age $a$. At time $t$, this probability is a function of the force of mortality from age 0 to age $x$. Denoting the force of mortality at age $a$ and time $t$ by $\mu(a, t)$, we can write the life table probability of surviving from birth to age $a$ as

$$
\ell_{p}(a, t)=\exp \left(-\int_{0}^{a} \mu(x, t) d x\right)
$$

The subindex $p$ in equations (1) and (2a) denotes that these are period measures. In the rest of the text, subscript $c$ will be used to identify cohort measures. For example, $\ell_{c}(a, t-a)$ is the life table probability of surviving from birth to age $a$ for the cohort born at time $t$ - $a$, i.e.

$$
\ell_{c}(a, t-a)=\exp \left(-\int_{0}^{a} \mu(x, t-a+x) d x\right) .
$$

and $e_{c}(0, t-a)$ is that cohort's life expectancy.

Here, it should be noted that at exact age $a$ and time $t$ the period and cohort force of mortality are equal therefore $\mu(a, t)$ does not have a subscript. However, this is not the case with the rest of the period and cohort measures. In contemporary low mortality countries there is a large difference between period and cohort life expectancies. For example, Goldstein and Wachter (2005) show that in the industrialized world the period life expectancy at year $t$ is approximately equal to the cohort life expectancy for persons born half a century ago, or $e_{p}(0, t+50) \approx e_{c}(0, t)$.

Specific methods to analyze change in life expectancy over time have been developed by various demographers. United Nations (1982), Pollard (1982, 1988), Arriaga (1984), Pressat (1985) and Andreev (1982; Andreev et al. 2002) focused on discrete differences in life expectancy between two periods of time. Keyfitz (1977, 1985) considered continuous change and derived a formula for the time-derivative of life expectancy. Mitra (1978), Demetrius (1979), Goldman and Lord (1986), Vaupel (1986), Hokkert (1987), Hill (1993) and Vaupel and Canudas-Romo (2003) further developed this approach. 
Several of these decomposition methods also offer the possibility of calculating age-specific contributions to the change in life expectancy. Thus, an interesting question to examine is how a change in mortality at a given age and time contributes to change in both period and cohort life expectancies. This leads to studying changes in life expectancies when the distribution and level of mortality are changing over time. To pursue these analyses, we follow some of the procedures developed in Vaupel and Canudas-Romo (2003).

\section{Period and cohort life expectancy age-decomposition}

We need to introduce two measures used in the developments that follow. Let the period probability density function describing the distribution of deaths (i.e., lifespans) in the life table population at age $a$ and time $t+a$ be denoted as $f_{p}(a, t+a)=\mu(a, t+a) \ell_{p}(a, t+a)$, and its cohort counterpart born $a$ years earlier as $f_{c}(a, t)=\mu(a, t+a) \ell_{c}(a, t)$. Another measure needed in the derivations to follow is the rate of progress in reducing death rates, defined as the derivative of the logarithm of the force of mortality, $\rho(a, t+a)=\frac{\partial \ln \mu(a, t+a)}{\partial t}$. In the rest of the text, a dot over a variable is used to denote the partial derivative with respect to time of that variable, e.g. $\rho(a, t+a)=\frac{\dot{\mu}(a, t+a)}{\mu(a, t+a)}$.

Vaupel and Canudas-Romo (2003) show that an age-specific contribution to the change in life expectancy is equal to the product of three components. These components are the rate of mortality improvement at that age, the remaining life expectancy at that age, and the mortality density function at that age. For the period life expectancy at birth at time $t+a$, the age-specific contribution of age $a$ denoted as $\dot{e}_{p, a}(0, t+a)$ is then

$$
\dot{e}_{p, a}(0, t+a)=\rho(a, t+a) e_{p}(a, t+a) f_{p}(a, t+a) .
$$

Adding those contributions over age gives the total change in period life expectancy at birth, $\dot{e}_{p}(0, t+a)$. Paralleling equation (3), it is possible to define an age-specific contribution to the change in cohort life expectancy. The ratio of period to 
cohort age-specific contributions, $\dot{e}_{p, a}(0, t+a) / \dot{e}_{c, a}(0, t)$, then allows us to see at which ages mortality changes affect cohort more than period life expectancy. Because the rate of mortality improvement $\rho(a, t+a)$, is the same in the period and cohort perspective, the ratio simplifies to

$$
\frac{\dot{e}_{p, a}(0, t+a)}{\dot{e}_{c, a}(0, t)}=\frac{\rho(a, t+a) e_{p}(a, t+a) f_{p}(a, t+a)}{\rho(a, t+a) e_{c}(a, t) f_{c}(a, t)}=\frac{e_{p}(a, t+a) f_{p}(a, t+a)}{e_{c}(a, t) f_{c}(a, t)} .
$$

Using the probability density functions describing the distribution of deaths, being $f_{c}(a, t)=\ell_{c}(a, t) \mu(a, t+a)$ for the cohort and $f_{p}(a, t+a)=\ell_{p}(a, t+a) \mu(a, t+a)$ for the period, we obtain

$$
\frac{\dot{e}_{p, a}(0, t+a)}{\dot{e}_{c, a}(0, t)}=\frac{e_{p}(a, t+a) \ell_{p}(a, t+a)}{e_{c}(a, t) \ell_{c}(a, t)} .
$$

As shown in equation (4) this ratio can also be expressed in terms of the ratio of the period over the cohort remaining life expectancy times the ratio of period to cohort survival function to that age. The expression on the right of equation (4) is a surprising result because the comparison does not contain our explicit measure of change, $\rho(a, t+a)$.

To gain an appreciation of how the period and cohort age-contributions differ, the following section presents a continuous model where mortality changes over age and time at constant rates.

\section{Changes in period and cohort life expectancy}

\subsection{Period and cohort models of mortality}

Model populations provide a useful way to examine age-specific contributions to changes in period and cohort life expectancy. The formulation used here is an extension of the Gompertz model of mortality where there is an infant mortality component and a continuous rate of decline over time. This model is a combination of the model 
proposed by Siler (1979) and the continuous rate of decline model discussed by Vaupel (1986) and Schoen et al. (2004). The force of mortality at age $a$ and time $t$ is defined as

$$
\mu(a, t)=A_{1} \exp \left[-B_{1} a-C_{1} t\right]+A_{2} \exp \left[B_{2} a-C_{2} t\right]+A_{3} \exp \left[-C_{2} t\right],
$$

where there are three constant terms which reflect the value of $\mu(0,0)=A_{1}+A_{2}+A_{3}$; parameters $B_{1}$ and $B_{2}$ that are fixed rates of mortality decline and increase over age, respectively, which account for infant and senescent mortality; and parameters $C_{1}$ and $C_{2}$ that are constant rates of mortality decrease over time. Parameters $A$ s and $B$ s come from the Siler model, while the $C$ s are used in Gompertz models with a continuous rate of decline (Vaupel, 1986 and Schoen et al., 2004). In the remaining text we refer to equation (5) as the Siler mortality change model.

In the model we begin with fairly high infant mortality (203 per thousand), resulting from the values of $A_{1}=0.2, A_{2}=0.0002$ and $A_{3}=0.003$. The early decline over age proceeds at a pace of $B_{1}=1$ with an overall increase with age at a rate of $B_{2}=0.1$. These values for parameters $A$ and $B$ have been adapted from a comparison of the Siler model with the different model life tables elaborated by Coale and Demeny (Gage and Dyke, 1986). At time 0, period life expectancy is 38.5. These values approach those observed in populations with historical data. For example, in Sweden in the year 1800, infant mortality was 227 per thousand and life expectancy 32.19 years. For the pace of mortality improvement we have chosen $C_{1}=0.015$ and $C_{2}=0.01$. These values correspond to a $1.5 \%$ decline at younger ages and mortality improvement of one percent at older ages. The decline at younger ages in several European countries occurred at an even faster rate (Woods et al., 1988 and 1989), and the rate of one percent is below the current average mortality decline in the West. 


\subsection{Trends, gaps and lags involving period and cohort life expectancies}

Figure 1 shows the period and cohort life expectancies for the Siler mortality change model in equation (5) over 600 years.

\section{Figure 1: Period and cohort life expectancy in a Siler mortality change model with rates of decline over time of $\mathrm{C} 1=0.015$ and $\mathrm{C} 2=0.01$}

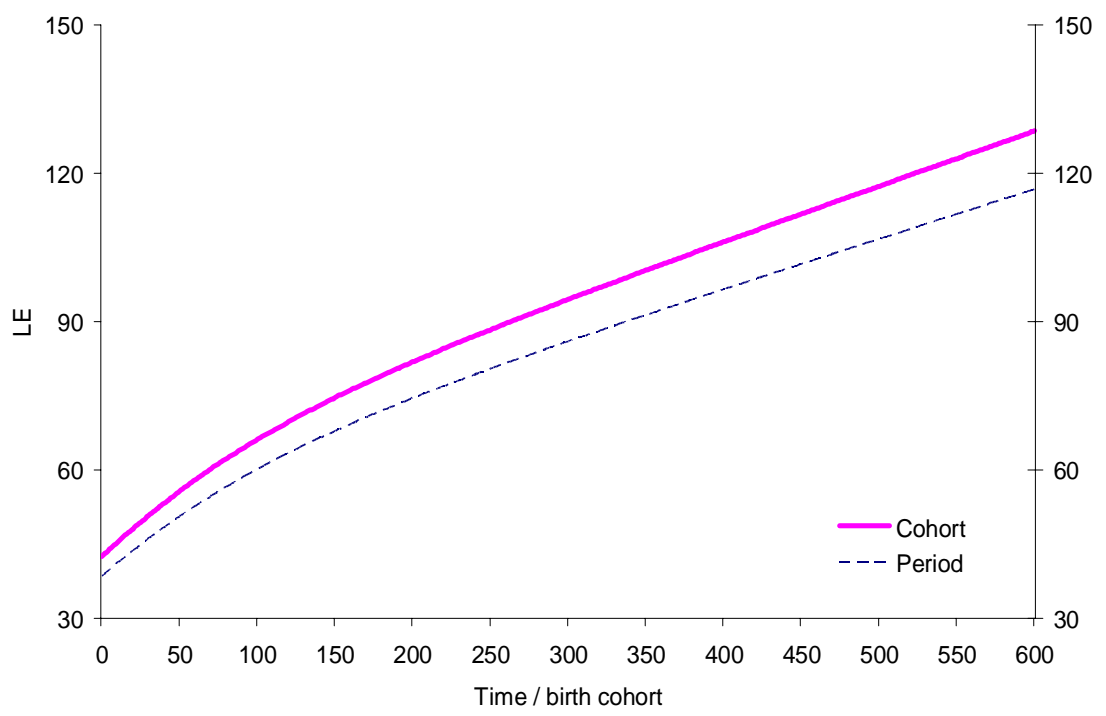

At time zero, the period and cohort life expectancies are 38.5 and 42.4, respectively. Over time both measures increase at an initially rapid but steady declining rate of increase. The initial fast pace of increase is related to infant mortality improvements. That can be seen from a Siler mortality change model with no senescent mortality improvement, $C_{2}=0$ (not shown here), which produces a similar trend in both life expectancies. As infant mortality falls to low levels, its impact declines toward zero.

In Figure 1, after 200 years the Siler mortality change model becomes a Gompertz model as changes in infant mortality are almost negligible. The increase in life expectancy becomes very close to the slopes determined by parameters $B_{2}$ and $C_{2}$. For period life expectancy, the slope is close to $C_{2} / B_{2}$. For the cohort LE, it is about 
$C_{2} /\left[B_{2}-C_{2}\right]$, so over time the change in the cohort measure is greater than its period counterpart (Schoen and Canudas-Romo, 2004).

Goldstein and Wachter (2005) studied gaps and lags between the period and cohort life expectancies in a model that allowed changes over time to vary at all ages. Here we focus on a simpler model that implies nearly linear time trajectories for the period and cohort life expectancies. The gap indicates the number of years at a given time between period and cohort life expectancy. For example, in our Figure 1 at time 100 the gap is about 6 years, while at time 450 it is about 10.1 years. In contrast, the time lag indicates the number of years between the time that cohort LE reaches a given level and the period LE attains that level. As shown in Diagram 1, from the estimated slopes for the period and cohort LE, and the gap values it is possible to obtain good estimates for the lags.

\section{Diagram 1: Relationship of the gaps and lags between period and cohort life expectancy}

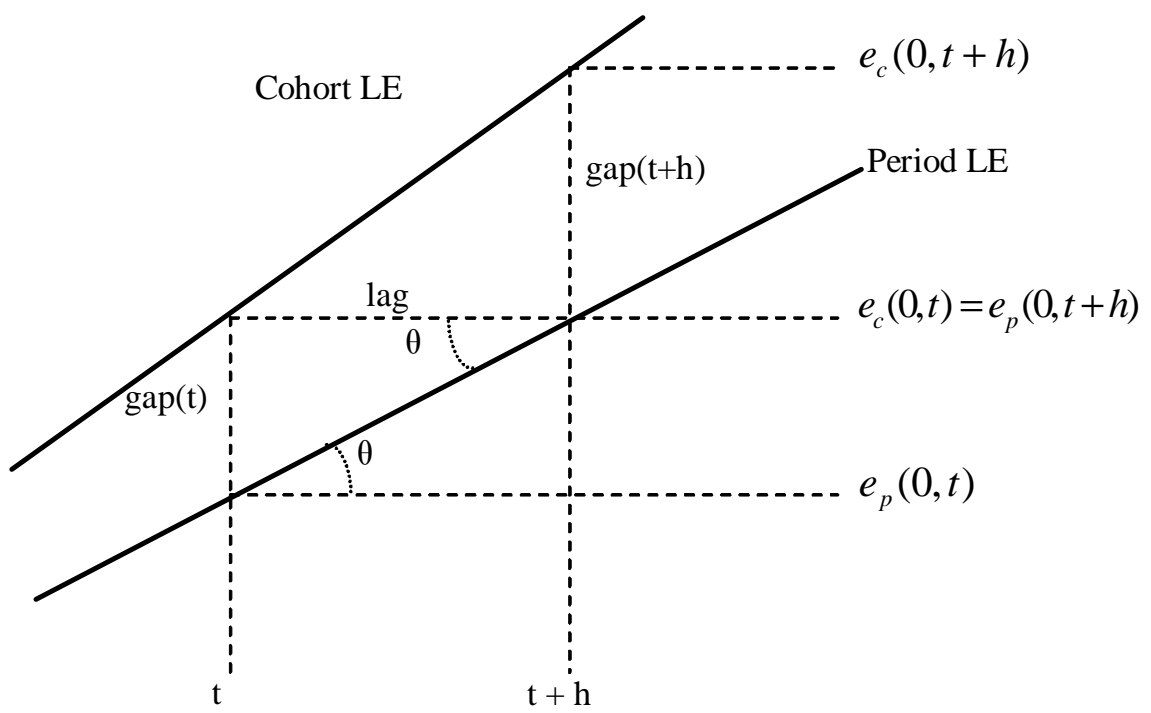

Diagram 1 shows that the gap between cohort LE and period LE at time $t$ is also equal to the difference between the period LE at time $t$ and at time $t+h$. The slope of the period life expectancy is equal to the tangent of the angle created between the LE curve 
and a horizontal line at the level of the period LE, $\tan (\theta)=\frac{C_{2}}{B_{2}}$. This is also the definition of the derivative with respect to time of this linear period life expectancy, $\dot{e}_{p}(0, t)=\tan (\theta)=\frac{C_{2}}{B_{2}}$. Two identical triangles are formed between the vertical lines at time $t$ and $t+h$, the horizontal lines at the levels of the period and cohort life expectancies at time $t$, and the curve of the period LE. The tangent of the angle can also be expressed in terms of the sides of the triangles, as the gap over the lag, $\tan (\theta)=\frac{\operatorname{gap}(t)}{\operatorname{lag}(t)}$. Therefore, the lag between cohort LE at time $t$ and period LE at time $t+h$ is equal to

$$
\operatorname{lag}(t)=\operatorname{gap}(t)\left(\frac{B_{2}}{C_{2}}\right)=\left[e_{c}(0, t)-e_{p}(0, t)\right]\left(\frac{1}{\dot{e}_{p}(0, t)}\right) .
$$

In Figure 1 at time 450, the gap is 10.1 and the lag is 101 years, which is exactly the result from applying equation (6) with our values of $C_{2}=0.01$ and $B_{2}=0.1$. A similar procedure can be carried out to find the gap at time $t+h$, using the slope of the cohort LE and the previous lag,

$$
\operatorname{gap}(t+h)=\operatorname{lag}(t)\left(\frac{C_{2}}{B_{2}-C_{2}}\right) .
$$

For example, the value of the cohort life expectancy at time 450 corresponds to the period life expectancy at time 551, a lag of 101 years. At time 551 the observed gap between the cohort and period life expectancy is 11.2 (= 123.1 - 111.9), which also is the result provided by equation (7).

From equations (6) and (7) and for our values of $C_{2}=0.01$ and $B_{2}=0.1$ the lag at time $t$ will be 10 times the gap observed at that time while the gap at time $t+h$ will be one-ninth of the $\operatorname{lag}(\mathrm{t})$.

Furthermore, it is possible to relate gaps at different points in time by going backwards substituting at each time the relations in equations (7) and (6). For example, the gap at time $t$ can be expressed in terms of the previous lag, $\operatorname{lag}\left(t-h_{1}\right)$, as shown in (7), and this lag can be substituted by the previous gap as shown in equation (6), 


$$
\operatorname{gap}(t)=\operatorname{gap}\left(t-h_{1}\right)\left(\frac{B_{2}}{C_{2}}\right)\left(\frac{C_{2}}{B_{2}-C_{2}}\right)=\operatorname{gap}\left(t-h_{1}\right)\left(\frac{B_{2}}{B_{2}-C_{2}}\right) .
$$

For our previous example, the gap at time 551 is 11.2 which is 1.11 times the previous gap at time 450 of 10.1 . These steps can be repeated to relate any two gaps. For example, if two gaps are separated by $n$ known lags we have the following relation

$$
\operatorname{gap}(t)=\operatorname{gap}\left(t-L_{n}\right)\left(\frac{B_{2}}{B_{2}-C_{2}}\right)^{n},
$$

where $L_{n}$ is the addition of the $n$ lags that separate the two gaps. This total lag is found as the addition of the individual $h$ lags $L_{n}=h_{1}+h_{2}+\ldots+h_{n}$. Similar calculations can be done to relate two lags based on known gaps.

To further analyze the disparity between the period and cohort life expectancy, the next section presents the examination of age-specific contribution to changes in these measures.

\subsection{Age-specific contributions to changes in period and cohort life expectancy}

Figures $2 \mathrm{ab}$ show the Lexis surfaces of the age-specific contributions to the change in period and cohort life expectancy in a Siler mortality change model.

The period and cohort patterns in Figure 2ab are very similar. Initially changes at early ages have a great impact on overall life expectancy, but that diminishes over time. As shown in Figure 1, this is also the time when both life expectancies increase the fastest. Once the infant contribution has vanished, the age-specific contributions increase over age to a maximum around the ages where most of the deaths are concentrated. For example, at year 400 (when the period life expectancy is 96.5) this maximum occurs between ages 90 and 130. Finally, at the very high ages there is a pronounced decline in impact. The peak of the distribution of age-contributions in life expectancy increases over time as the death distribution moves to older ages. During this second phase of the model where mainly senescent mortality is taking place, increments in life expectancy are slower. 
Figure 2: Age-contributions to the change in the period life expectancy (a) and in the cohort life expectancy (b) per thousands, Siler mortality change model with two rates $\mathrm{C} 1=0.015$ and $\mathrm{C} 2=0.01$

(a)

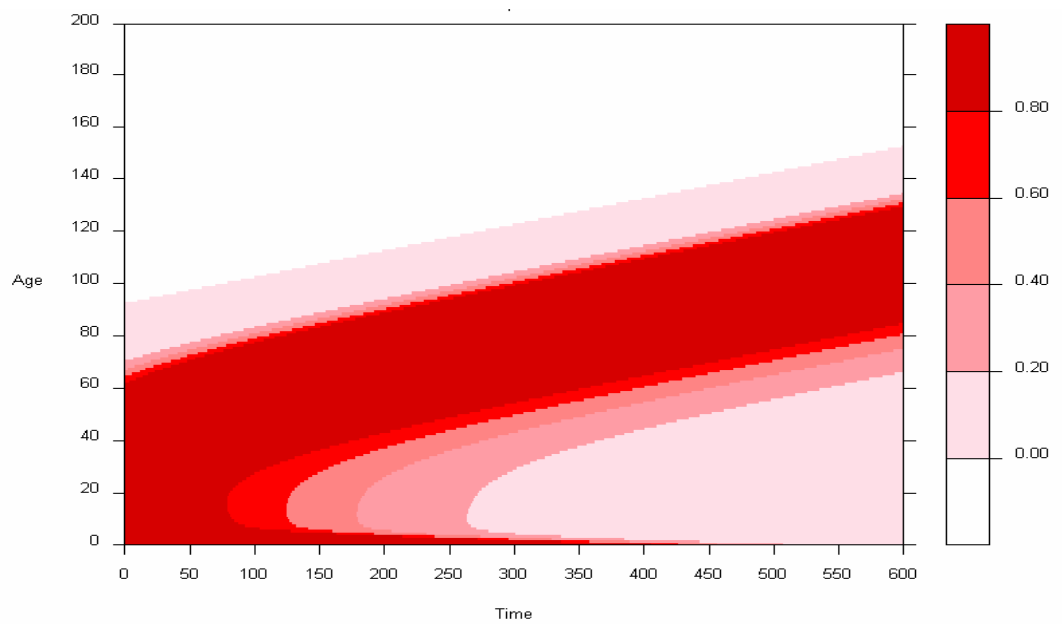

(b)

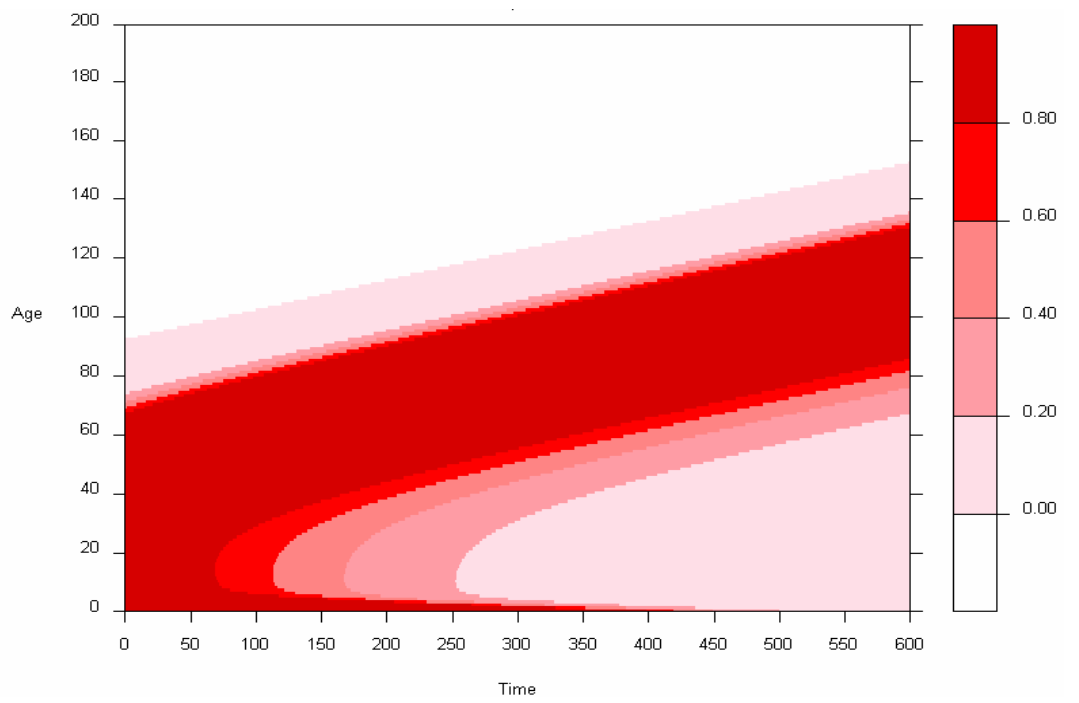


Figure 3 depicts the Lexis surface for the ratio of (i) the age-specific contributions to the change in period life expectancy over (ii) the corresponding age-specific contributions to the change in cohort life expectancy.

\section{Figure 3: Ratio of age-specific contributions to the change in the period over change in the cohort life expectancy, Siler mortality change model with rates of decline over time $\mathrm{C} 1=0.015$ and $\mathrm{C} 2=0.01$}

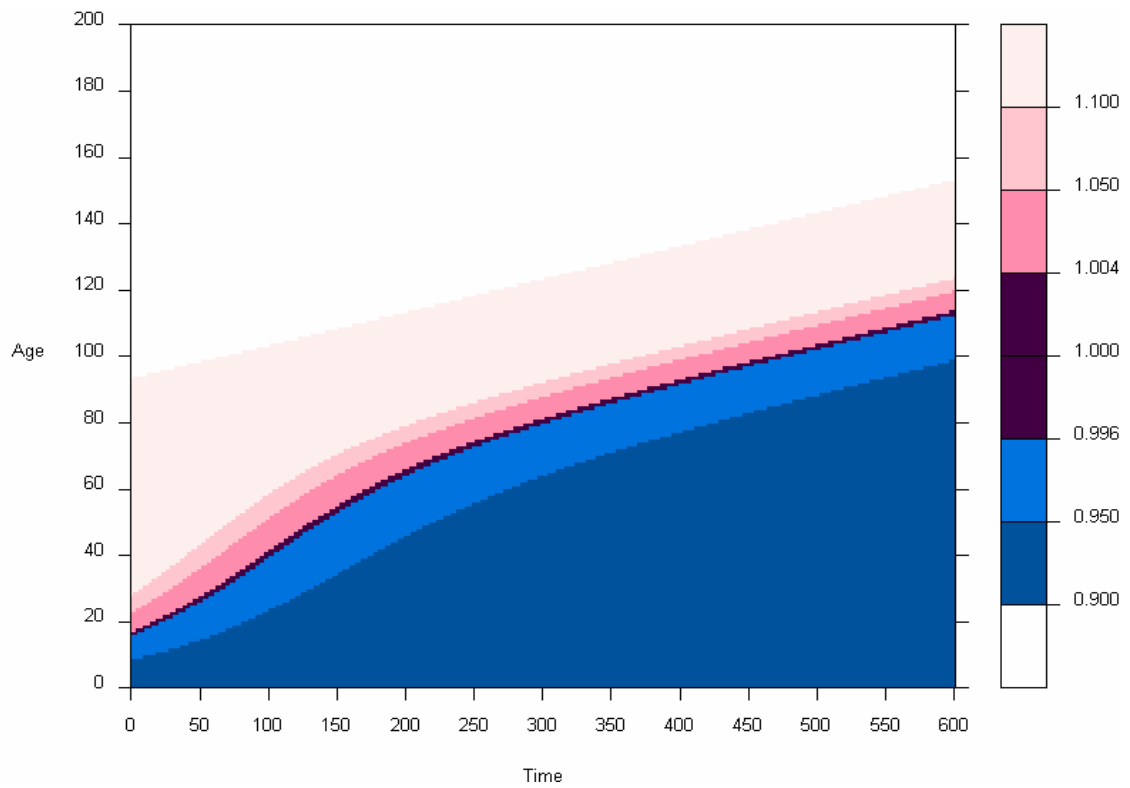

Figure 3 shows the steady increase over time in the age at which the ratio is 1 . In the early years, the ratio is above 1 at most ages. That indicates that most ages make greater age-contributions to the period LE as infant mortality plays a larger role. At time 600 , most of the ratios are below 1, showing that cohorts benefit more over a wide range of ages. However, at year 600 period LE is 116.8 and it is at advanced ages where most of the improvements in mortality are occurring. This increase in the age at which the ratio is one parallels the increase of life expectancies in Figure 1, with an accelerated pace at the beginning and a slower pace later.

To further analyze the age pattern of this ratio, we re-examine equation (4). The ratio of age-contributions can be separated into two terms reflecting past and future period and cohort experience, i.e. 


$$
\frac{\dot{e}_{p, a}(0, t+a)}{\dot{e}_{c, a}(0, t)}=\left[\frac{\ell_{p}(a, t+a)}{\ell_{c}(a, t)}\right]\left[\frac{e_{p}(a, t+a)}{e_{c}(a, t)}\right] .
$$

Higher mortality in the past causes fewer cohort survivors to age $a, \ell_{c}(a, t)$, than in the corresponding period, $\ell_{p}(a, t+a)$, implying numbers always above 1 for this measure of the past. Contrary to this is the ratio of remaining life expectancy, where cohort values are higher than period, indicating a ratio always below 1 for this measure of the future.

Figure 3 shows that at any given time the ratio increases with age. For younger ages it is below one, indicating that changes at those ages have more impact on cohort than period life expectancy. With mortality declining over time, young cohorts at time $t$ will experience death rates at older ages lower than those experienced by older persons at time $t$, while differences in survivorship remain modest. Therefore, in equation (4) the ratio of period to cohort LE dominates the ratio of period to cohort survivorship. The inverse process occurs at older ages where the values of the ratio are above one. The cohorts that reach advanced ages at time $t$ have experienced death rates at earlier ages greater than those seen in the period $t$, while the period to cohort LE ratio is modest.

Until now we have discussed results of changes in mortality occurring over periods. However, the relations found here also hold in the case of changes occurring over cohorts, as verified by analyses of model populations (not shown). Furthermore, similar results are obtained if instead of the senescent mortality term in equation (5) we include a logistic model as those studied by Bongaarts (2005). 


\section{Examining period and cohort mortality in Sweden}

To assess age-specific contributions to period and cohort life expectancy in Sweden, we use data derived from the Human Mortality Database (2004). To calculate the agespecific contributions to cohort life expectancy for cohorts that are not extinct we have extrapolated future mortality. That was done using a continually declining mortality model, similar to that in equation (5), with a constant pace of decline of $C=0.01$. For example, if the last year with available data is 2002, then the age-specific death rates for that year, $\mu(a, 2002)$ are the base values for the model. For age $a$ and year $t>2002$ the force of mortality is

$$
\mu(a, t)=\mu(a, 2002) e^{-C(t-2002)} .
$$

Figure 4 presents the period and cohort life expectancies in Sweden 1751-2002 with extrapolated cohort values for the years 1912 to 2002 using the death rates given by equation (9).

Figure 4: Cohort and period life expectancy, with cohorts completed with a continuous declining pace in mortality of $\mathrm{C}=\mathbf{0 . 0 1}$, Sweden

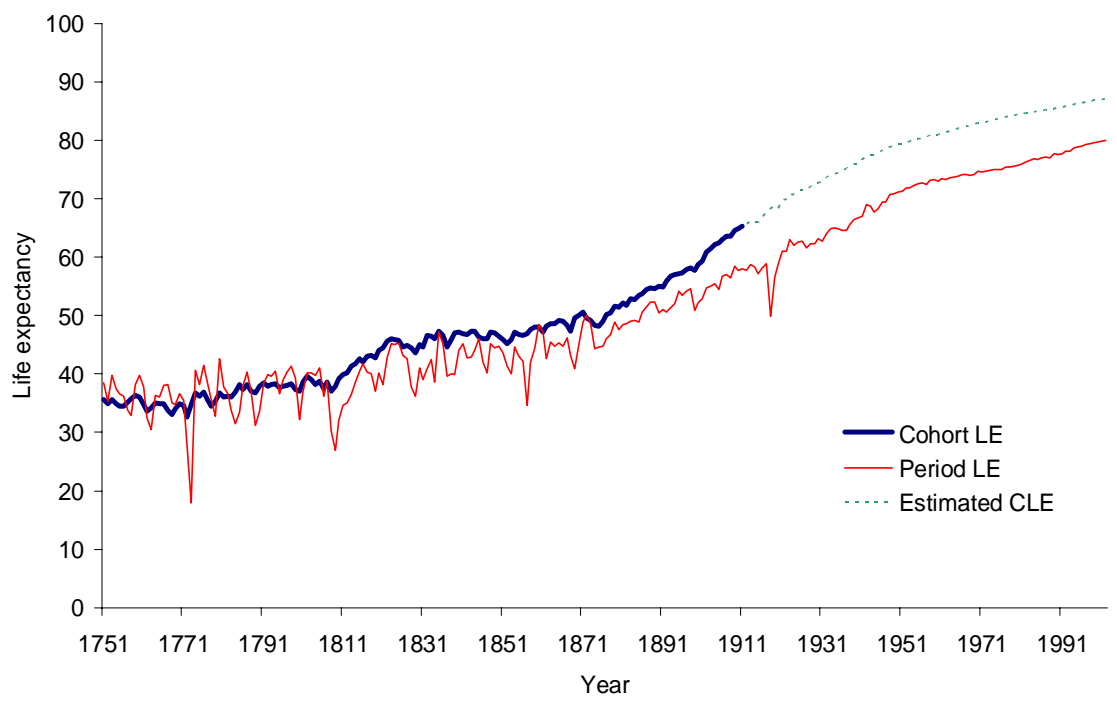


During the last years of the twentieth century the fluctuations observed in the period measure have largely disappeared. The estimated values of the cohort life expectancy, with the selected decline over time, correspond to a gap of around 7 years between the two life expectancies for 1911. Before 1900, however, that gap was much smaller and more erratic.

The gap observed between period and cohort life expectancies begins at the middle of the nineteenth century in Sweden. This is also the period of great improvement in infant mortality which contributes to the increase in both life expectancies, but more marked for the cohort measure. From about 1880 on, the pattern in Figure 4 resembles that in Figure 1.

Equations (6)-(8) show the relations between gaps and lags in a simple mortality model. These equations can be used for Sweden assuming that life expectancy moves linearly with a constant slope. This linear assumption has been shown to be realistic in developed countries over the last half of the 20th century (White, 2002). For Sweden, in 1911, the last year with full information on cohorts, the period and cohort life expectancies were 58.0 and 65.2 respectively. The gap between period and cohort life expectancies was thus 7.2 years. Calculating the derivative as the average of all annualized changes in period life expectancy from 1911 to each year from 1912 through 1950 we obtain an average slope of 0.30. Applying equation (6), a lag of 24 years is found. Comparing the cohort life expectancy of 65.3 in 1911 with 64.9, the period LE in year 1935, gives a discrepancy of only 0.4 years.

Period life expectancy in the industrialized world has followed a linear trend during the second half of the twentieth century so equation (6) should give a good approximation of the lags implied by the observed gaps (Goldstein and Wachter, 2005). For example, in 1950 cohort life expectancy was 79.5 years, with period life expectancy 8.4 years lower. For the year 1999, period life expectancy is 79.5 , reaching the cohort value of 49 years earlier. With the slope of period life expectancy calculated as 0.18 , equation (6) gives a lag of 46.7 years, which is 2.3 years below the actual lag of 49 years. However, the period life expectancy for 1997, the estimated year, was 79.3, giving an error in the lag of only 0.2 years.

For Sweden between 1900 and 2002, Figure 5 shows the Lexis surface for the ratio of the age-specific contributions to period relative to cohort life expectancies, as calculated from equation (4). 
Figure 5: Ratio of the period over the cohort age-contribution, Sweden 19002002. Cohorts completed with a pace of decline over time $\mathrm{C}=0.01$

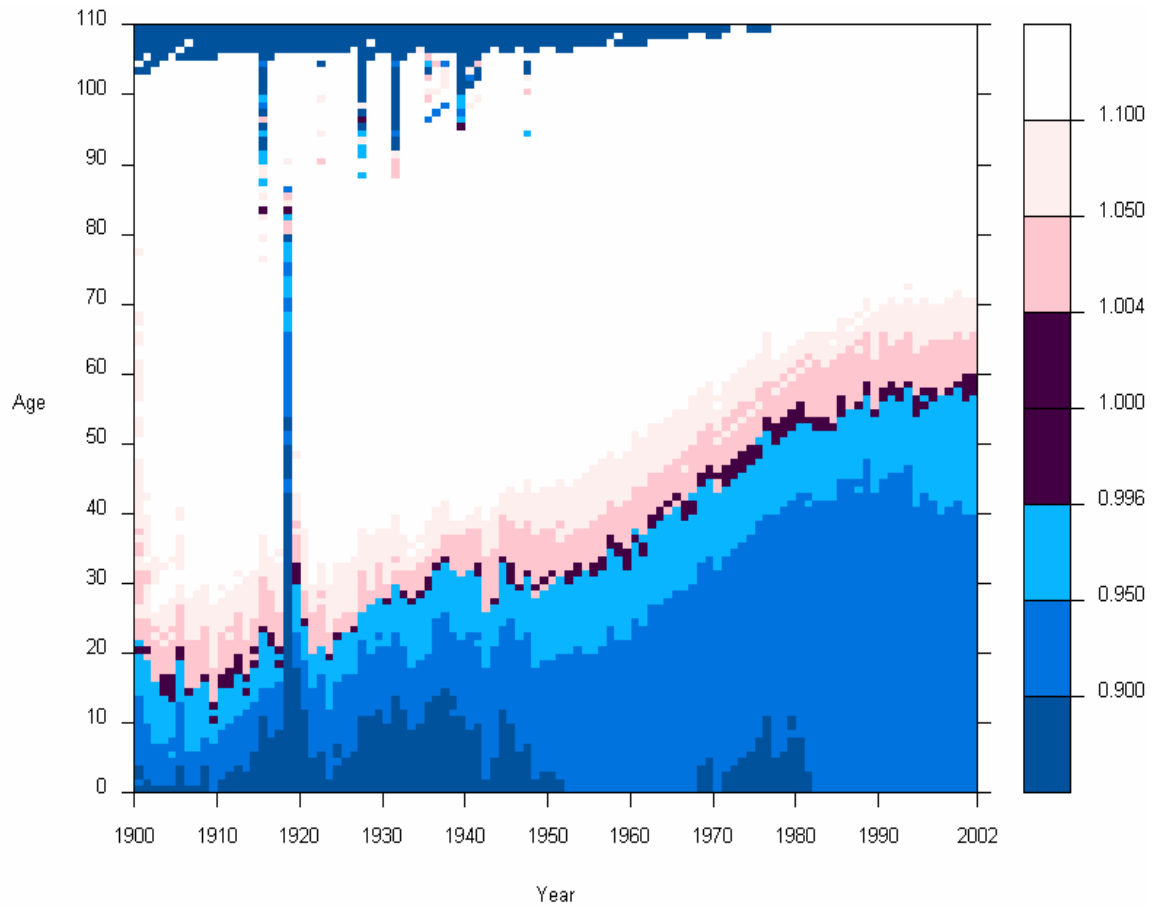

Results similar to the Siler mortality change model of Figure 3 can be seen in Figure 5, specially after 1940. The young ages make greater contributions to cohort LE while at older ages the period LE gains more. There is also a clear upward time trend in the crossover value of 1 . At the beginning of the twentieth century the value of one is found at age 20, but that rises to age 60 at the end of the century. There is an interruption of the pattern during the Spanish flu pandemic in 1918. The sharp rise in mortality during that year impacted the period much more than any cohort, and led to greater cohort gains in LE up into high ages. 


\section{Conclusions}

The disparity between period and cohort life expectancy can be measured at a given time or over the number of years it takes for period LE to attain the cohort level. For our continuously declining mortality model these two measures are linked by a simple relationship. For the industrialized world, where countries have experienced linear trends in life expectancy in the last half century, a similar though approximate relationship exists. While the cohort-period gap is fairly small and grows slowly over time, the lag is sizeable and grows much more rapidly.

To further analyze the dynamics of the two life expectancies we examine the agespecific contribution of changes in death rates to period and cohort life expectancy. Cohort life expectancy gains more from changes at younger ages, while the period measure gains more at older ages. The ratio of the contributions to period over cohort life expectancy can be seen as the product of two terms accounting for past and future changes in mortality. At the age when past mortality experience is balanced by future changes the ratio is one. As infant mortality falls to low levels improvements are concentrated at older ages, and the balancing age rises.

The present pattern of mortality change suggests widening cohort-period gaps and especially lags, as experience at more and more ages contributes more to cohort LE than to period LE.

\section{Acknowledgments}

The first author gratefully acknowledges support from the DeWitt Wallace postdoctoral fellowship awarded by the Population Council. The authors thank the two anonymous referees for their helpful comments. 


\section{References}

Andreev, Evgueni M. 1982. "Method Komponent v Analize Prodoljitelnosty Zjizni." [The Method of Components in the Analysis of Length of Life]. Vestnik Statistiki 9:42-47.

Andreev, Evgueni M., Vladimir Shkolnikov and Alexander Z. Begun. 2002. "Algorithm for Decomposition of Differences Between Aggregate Demographic Measures and its Application to Life Expectancies, Gini Coefficients, Health Expectancies, Parity-Progression Ratios and Total Fertility Rates." Demographic Research 7:499-522.

Arriaga, Eduardo E. 1984. "Measuring and Explaining the Change in Life Expectancies." Demography 21:83-96.

Bongaarts, John and Griffith Feeney. 2002. "How Long Do We Live?" Population and Development Review 28(1):13-29.

Bongaarts, John. 2005. "Long-Range Trends in Adult Mortality: Models and Projection Methods." Demography 42(1):23-49.

Demetrius, Lloyd. 1979. "Relations Between Demographic Parameters." Demography 16:329-338.

Gage, Timothy and Bennett Dyke. 1986. "Parameterizing Abridged Mortality Tables: The Siler Three-Component Hazard Model." Human Biology 58(2):275-291.

Goldman, Noreen and Graham Lord. 1986. "A New Look at Entropy and the Lifetable." Demography 23:275-282.

Goldstein, Joshua R. and Kenneth W. Wachter. 2005. "Gaps and Lags: Relationships Between Period and Cohort Life Expectancy." Unpublished Manuscript, Princeton University and University of California, Berkeley.

Hill, Gerry. 1993. "The Entropy of the Survival Curve: An Alternative Measure." Canadian Studies in Population 20:43-57.

Hokkert, Ralph. 1987. "Lifetable Transformations and Inequality Measures: Some Noteworthy Formal Relations." Demography 24:615-622.

Human Mortality Database. University of California, Berkeley (USA), and Max Planck Institute for Demographic Research (Germany). Available at www.mortality.org or www.humanmortality.de (data downloaded on [5/1/04]). 
Keyfitz, Nathan. 1977. "What Difference Does it Make if Cancer Were Eradicated? An Examination of the Taeuber Paradox." Demography 14:411-418.

---. 1985. Applied Mathematical Demography. 2nd ed. New York: Springer.

Mitra, S. 1978. “A Short Note on the Taeuber Paradox.” Demography 15:621-623.

Pollard, J. H. 1982. "The Expectation of Life and its Relationship to Mortality." Journal of the Institute of Actuaries 109:225-240.

---. 1988. "On the Decomposition of Changes in Expectation of Life and Differentials in Life Expectancy." Demography 25:265-276.

Pressat, Roland. 1985. "Contribution des écarts de Mortalité par Age la Différence des Vies Moyennes." Population 4-5:765-770.

Preston, Samuel, H., Patrick Heuveline and Michel Guillot. 2001. Demography: Measuring and Modeling Population Processes. Oxford: Blackwell Publishers.

Schoen, Robert and Vladimir Canudas-Romo. 2004. "Changing Mortality and Average Cohort Life Expectancy." Paper presented at the workshop on Tempo Effects on Mortality, November 18-19, New York.

Schoen, Robert, Stefan H. Jonsson and Paula Tufis. 2004. "A Population with Continually Declining Mortality." Working Paper 04-07, Population Research Institute, Pennsylvania State University, University Park, PA.

Siler, William. 1979. "A Competing-Risk Model for Animal Mortality." Ecology 60(4):750-757.

United Nations. 1982. Levels and Trends of Mortality Since 1950, Study 74. New York: United Nations; Dept. of International Economic and Social Affairs.

Vaupel, James W. 1986. "How Change in Age-Specific Mortality Affects Life Expectancy." Population Studies 40:147-157.

Vaupel, James W. and Vladimir Canudas-Romo. 2003. "Decomposing Change in Life Expectancy: A Bouquet of Formulas in Honor of Nathan Keyfitz's $90^{\text {th }}$ Birthday." Demography 40(2):201-216.

White, KM. 2002. "Longevity Advances in High-Income Countries 1955-96." Population and Development Review 28(1):59-76.

Woods, RI, PA Watterson and JH Woodward. 1989. "The Cause of Rapid Infant Mortality Decline in England and Wales, 1861-1921. Part II" Population Studies 43(1):113-132. 
Canudas-Romo \& Schoen: Age-specific contributions to changes in the period and cohort life expectancy

Woods, RI, PA Watterson and JH Woodward. 1988. "The Cause of Rapid Infant Mortality Decline in England and Wales, 1861-1921. Part I." Population Studies 42(3):343-366. 